\title{
MINIMAL SUPERIOR ARTICULAR PROCESS REMOVAL OF FACET JOINT IN LATERAL INTERPEDICULAR APPROACH COULD PROVIDE A BETTER EXPOSURE IN FAR LATERAL DISC HERNIATION TREATMENT: A TECHNICAL REPORT
}

\author{
(1) Halil CAN 1 , ๑ Atilla KIRCELLi² \\ ${ }^{1}$ Biruni University Faculty of Health Sciences, Department of Neurosurgery, Istanbul, Turkey \\ ${ }^{2}$ Başkent University, Department of Neurosurgery, Istanbul, Turkey
}

Objective: To assess the results of 28 patients who underwent lateral interpedicular surgical approach (LISA) and to compare the outcomes with the current literature.

Materials and Methods: Twenty-eight patients with far lateral lumbar disc herniation (FLLDH) undergoing LISA between 2015 and 2018 were retrospectively analyzed. Extruded or sequestered far lateral lumbar disc herniations, which cause radiculopathy, were included in this study. A visual analogue scale (VAS) and patient's subjective comment on the result of surgery with Mac Nab Classification were recorded at the pre- and post-operative follow-ups.

Results: There were 15 males and 13 females. The mean age was 50.5 \pm 9.65 years. Two patients had $L 2-3$ herniations, 11 had $L 3-4$, and 15 had L4-5. The mean duration of operation was $48.8 \pm 8.7$ minutes. Preoperative VAS scores $(9.32 \pm 0.61)$ were found to decline to $0.78 \pm 0.57$. The Mac $\mathrm{Nab}$ Classification of the postoperative $6^{\text {th }}$ month results yielded $78.5 \%$ to be excellent, $14.2 \%$ to be good and $7.1 \%$ to be fair. There were no complications, including CSF leak, nerve injury or hematomas.

Conclusion: The LISA is a minimally invasive, safe and simple procedure for FLLDH surgery with short hospital stay and duration of operation and with low complication rates.

Keywords: Far lateral disc herniation, lateral interpedicular approach, superior articular process, facet joint

\section{INTRODUCTION}

A far lateral lumbar disc herniation (FLLDH) is building of the disc material into the area that is lateral to the superior and inferior pedicles ${ }^{(1)}$. A FLLDH causes exiting nerve root compression, contrary to paramedian discs, which compress the nerve root at the level below ${ }^{(2)}$. Far lateral compartment is delineated as the area lateral to the superior and inferior pedicles, where the disc is located anteriorly, leading edge of the superior articular facet medially and the facet joint posteriorly ${ }^{(3-5)} \cdot 7-12 \%$ of all lumbar disc herniations are found to be FLLDH(6-8). Postacchini and Montanaro(9) defined the disc herniations lateral to the pedicle as "extreme lateral disc herniations, which is also used by Fankhauser and Trilobet ${ }^{(10,11)}$ however, some authors prefer the term "extraforaminal"(9-13). In recent studies, lateral disc herniations have been referred to as FLLDHs (2).

Macnab ${ }^{(14)}$ reported two cases of extraforaminal L5-S1 disc herniations leading L5 root compression, following a failed exploration at the L4-5 level in 1971. In 1974, Abdullah et al. ${ }^{(15)}$ described the extreme lateral lumbar disc herniations for the first time.

Clinical characteristics of FLLDHs differentiated from paramedian disc herniations, such as sharper radicular pain due to direct compression of the dorsal root ganglion and acute onset $^{(16)}$. Compression of the exiting nerve root and dorsal root ganglion causes some clinical symptoms ${ }^{(1)}$. Compared to paramedian disc herniations, FLLDHs are more prone to be at the upper lumbar levels and to have adjacent pathologies like paramedian or foraminal disc herniations and spinal stenosis at the same level ${ }^{(16)}$.

This study aims to present anatomical landmarks of the lateral interpedicular approach without opening the intertransverse fascia, as well as the route followed, and to ensure that this surgical approach becomes safer via identifying exiting root and dorsal root ganglion earlier. Accordingly, figures obtained from out fresh cadaver dissections and our clinical experiences of 28 cases were presented in this study. 


\section{MATERIAL AND METHODS}

\section{Patients and Methods}

For this research, 28 patients with FLLDHs undergoing lateral interpedicular (lateral micro neurosurgical) surgical approaches (LISA) between 2015 and 2018 were retrospectively analyzed. This study was a single-center analysis and all operations were done with informed consent of the patients. This is a retrospective study performed in accordance with Helsinki Declarations and it was reported from patients' files. A detailed neurological examination was performed in each patient with FLLDHs confirmed by a neuroradiological imaging.

Inclusion criteria were defined as:

- At least one month of severe leg pain (with or without low back pain),

- A radiologically documented extruded or sequestered far lateral disc herniation with or without foraminal component,

- Positive straight-leg raising or femoral stretch test upon neurological examination,

- Having motor or/and sensory deficit.

We excluded patients with tumors, infections, bleeding disorders, and L5-S1 disc herniations due to high iliac crest.

A visual analogue scale (VAS) was performed pre- and postoperatively in order to evaluate pain. Patient's subjective opinion was categorized as excellent (no pain), good (some pain), fair (moderate pain) or poor (unchanged or worse) depending on the MacNab classification. This study was approved by The Council of Forensic Medicine (decision no: ATK 0.01.00.08/74).

\section{Surgical Technique}

In the operating room, after attaching the patient to monitoring equipment and placing an intravenous catheter, the patient was anesthetized and fixed in a prone position. C-arm fluoroscopic guidance of the lumbar level was conducted, sterile draping was applied, and a 3-5 cm midline-vertical skin incision was made. Paravertebral muscle fascia was cut along the midline and blunt dissection of the paravertebral muscles was done. In order to expose the junction of the upper and lower facets, two thin Taylor retractors were then placed, one on the facet where the herniation was located and the other on the facet above. The inferior and superior facet joints and pars interarticularis were visualized under a surgical microscope (Figure 1).

First, a minimal bone resection was made from lateral to medial, at the inferior aspect of pars interarticularis, using a Kerrison rongeur. Then, the approach proceeded to the superior of pars interarticularis and inferior aspect of pars-facet junction. Minimal bone removal of superior articular process of the facet provided better exposure of the disc space (Figure 2). Minimal bone resection to recognize the root was performed at the inferior facet joint of the upper vertebrae and very limited bone resection was done at pars-facet joint junction of the inferior vertebrae (Figure 3). Visualization of the lateral aspect of the facet joint and transverse process, as well as the intertransverse muscle and intertransverse fascia, was not needed.

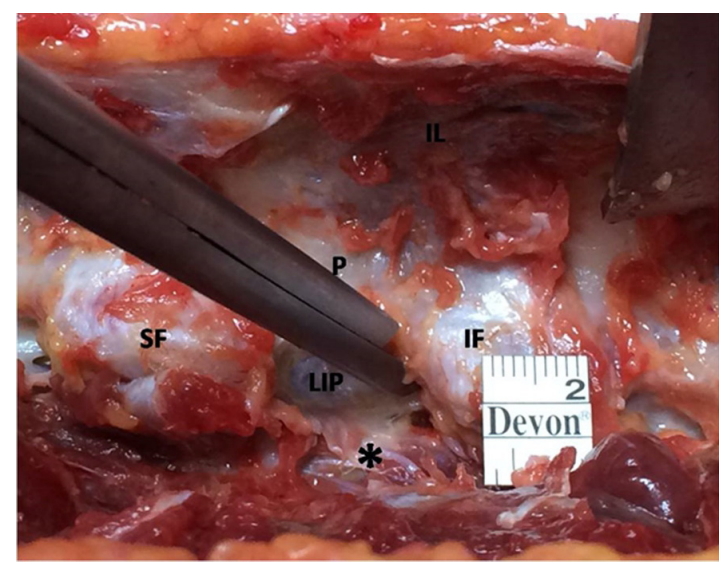

Figure 1. A picture from fresh cadaveric dissection. The inferior facet joint, superior facet joint, and pars interarticularis were exposed

SF: Superior facet joint, IF: Inferior facet joint, P: Pars interarticularis, IL: Interlaminar area, LIP: Lateral interpedicular area, ${ }^{*}$ : Intertransverse muscle and fascia

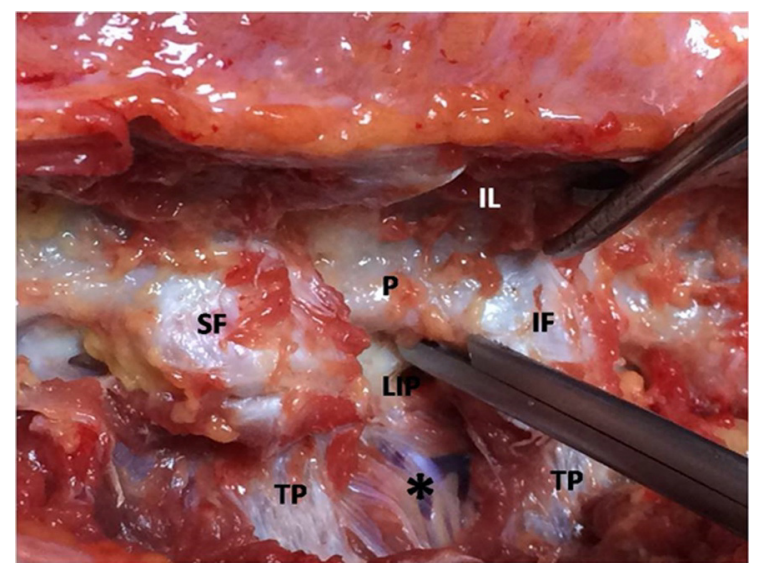

Figure 2. A picture from fresh cadaveric dissection. A minimal bone resection was performed from the lateral inferior aspect of the pars interarticularis to the medial aspect using a Kerrison rongeur

SF: Superior facet joint, IF: Inferior facet joint, TP: Transverse process, P: Pars interarticularis, IL: Interlaminar area, LIP: Lateral interpedicular area, ${ }^{*}$ : Intertransverse muscle and fascia

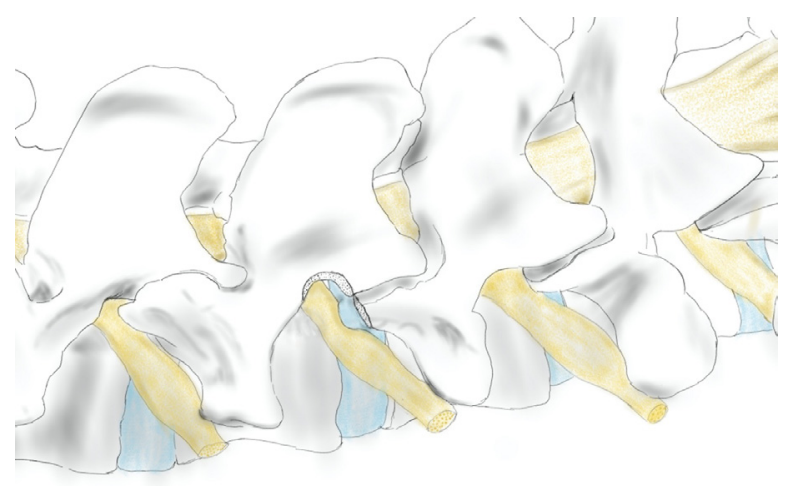

Figure 3. Pictogram, bone removal of superior articular facet and pars interarticularis 
turkishspine

At the beginning of the surgery, exiting nerve root at the medial aspect of the upper pedicle at the same level, was exposed via limited resection of the inferior facet joint of the upper vertebrae (Figure 4).

Then, the exiting nerve root was palpated at the level where it exited, using a blunt nerve hook. Subsequently, exiting nerve root was completely exposed to the resection of pars-facet joint junction of the inferior vertebrae. Since there was no need to visualize lateral aspect of the facet joint and transverse processes, intertransverse muscle and fascia were not opened (Figure 1, 2, 4).

Veins, that were located at the medial aspect of the exiting nerve root, were coagulated with bipolar cautery. Disc fragment, sequestered or extruded, were palpated using a blunt nerve hook and removed (Figure 5).

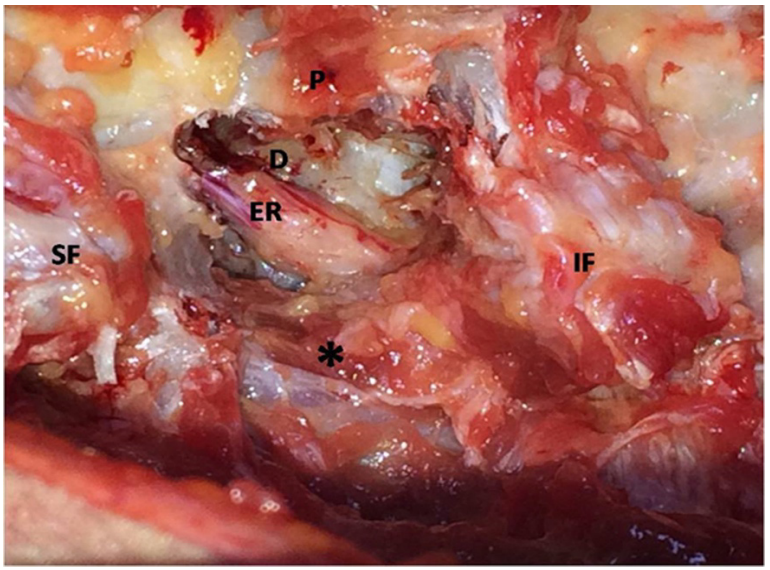

Figure 4. A picture from fresh cadaveric dissection. The nerve root exiting from the medial aspect of the upper pedicle was exposed via minimal bone resection at the superior and inferior facet joint and pars interarticularis

SF: Superior facet joint, IF: Inferior facet joint, P: Pars interarticularis, ER: Exiting nerve root, D: Intervertebral disc, ${ }^{*}$ : Intertransverse muscle and fascia

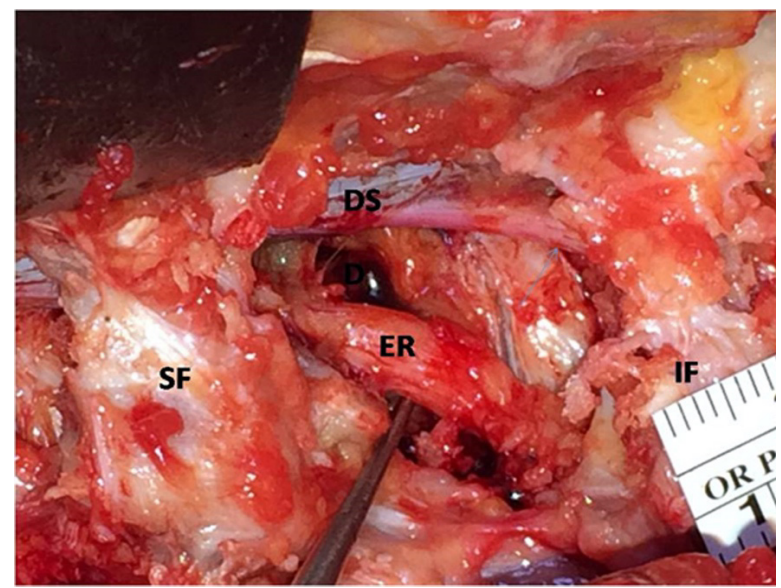

Figure 5. A picture from fresh cadaveric dissection. Pars interarticularis is completely removed

SF: Superior facet joint, IF: Inferior facet joint, blue arrow: Traversing root, ER: Exiting nerve root, D: Intervertebral disc, DS: Dural sac, ${ }^{*}$ : Intertransverse muscle and fascia
Entering the disc space, bone removal was made laterally through medial aspect of pars and caudally from the superior articular process of the inferior vertebra using a Kerrison rongeur. Following the incision of the posterior longitudinal ligament, far lateral disc fragments with foraminal components were removed. A discectomy was performed and hemostasis was achieved in all patients, who were then closed up and extubated. Each patient was monitored in the unit during the early postoperative period, mobilized at the same day and discharged the following day.

All patients were assessed pre- and postoperatively for pain according to a VAS and the postoperative MacNab criteria.

\section{Statistical Analysis}

SPSS v21 for Windows (IBM Corp., Armonk, NY, USA) was used to analyze our data. Means \pm standard deviations were used for normally distributed continuous variables [ $p>0.05$ in Kolmogorov-Smirnov test or Shapiro-Wilk $(\mathrm{n}<30)]$ and paired $T$ test was used to compare them. Non-normally distributed variables were defined as medians and compared using Kruskal-Wallis test. To investigate the relationship between the factors, Spearman's rank correlation coefficient was used. A p value below 0.05 was regarded as statistically significant.

\section{RESULTS}

This study included 28 retrospectively analyzed patients with FLLDHs. Out of 28 patients, two underwent surgery for FLLDHs at L2-3, 11 at L3-4 and 15 at L4-5 levels. Fifteen were male and 13 were female, with a mean age of $50.6 \pm 9.67$ years. The mean duration of complaints was 5.7 weeks. Neurological examination of the patients yielded, positive femoral nerve stretch test in $82.1 \%$ and positive Laseque's sign in $10.7 \%$. Seven point one percent of the patients had both tests positive. Overall, $67.8 \%$ had motor deficits, whereas $78.5 \%$ had sensory deficits.

Preoperative magnetic resonance imagings revealed that $46.6 \%$ of the patients had both far lateral disc herniations and foraminal fragments, while $57.6 \%$ had only far lateral disc herniation. Forty-six point four percent underwent sequestrectomy, 32.1\% sequestrectomy and discectomy, and $21.4 \%$ discectomy. The mean operation surgery was $48.8 \pm 8.7$ minutes.

There was no nerve root injury, cerebrospinal fluid leak, hematomas or infections at the operation site. At the $6^{\text {th }}$ month follow-up assessment, VAS score was found to decline from $9.32 \pm 0.61$ to $0.78 \pm 0.57 \quad(p<0.0001)$. MacNab classification evaluation showed the patient satisfaction to be excellent in $78.5 \%$, good in $14.2 \%$, and fair in $7.1 \%$. A previously existing dysesthesia progressed in one patient and was managed with medical treatment. There was no segmental instability on the postoperative $6^{\text {th }}$ month lumbar computed tomography scans (Figure 6). 


\section{DISCUSSION}

Abdullah et al. ${ }^{(15)}$ reported the clinical characteristics of FLLDHs in order to distinguish them from paramedian disc herniations for the first time in 1974. At that point, neurosurgeons were less familiar with FLLDHs since they could not be demonstrated via myelography or due to limited exploration. Following the evaluation of neuroradiological modalities, FLLDHs with or without foraminal components become more well-known ${ }^{(17)}$.

FLLDHs constitute $7-12 \%$ of all disc herniations that are located in lumbar area ${ }^{(6-8)}$. Associated radicular leg pain is due to direct compression of both exiting nerve root and the dorsal root ganglion ${ }^{(7)}$. Park et al.(16) suggested that radicular leg pain was more severe in FLLDHs compared to paramediandis herniations. They also reported that this phenomenon was associated with more acute onset of symptoms before surgery (64 days vs 31 days). In our study, patients with extruded discs which were migrated superolaterally into the neural foramen were found to have shorter duration of symptoms when compared to those without migration. This was explained with the fact that migrated discs caused more direct irritation of the dorsal root ganglion.

In a study of over 200 cases of FLLDH, including cadaver dissections, Schlesinger et al. ${ }^{(18)}$ suggested that craniocaudally the bone amount that had to be removed increased while working space decreased. They also reported, in the lower levels, the disc space was located more inferior than the attentive facet joint compared to upper levels. Therefore, an increased amount of bone had to be removed from the superolateral aspect of the facet joint and pars interarticulars, thus the dorsal root ganglion could be easily visualized with this exposure ${ }^{(18)}$. Similarly, in our clinical series and cadaver dissections, there was an increased amount of bone and the far lateral compartment and disc space were overlaid by an increased quantity of bone (Figure 7). In addition, there was decreased working space from L1 to L5.

Porchet et al. ${ }^{(17)}$ reported that motor deficit was a more reliable finding than dysesthesia in FLLDH. However, Park et al.(16) reported that sensory dysesthesia in FLLDH was more remarkable than a motor deficit.Viswanathan et al. ${ }^{(13)}$ suggested

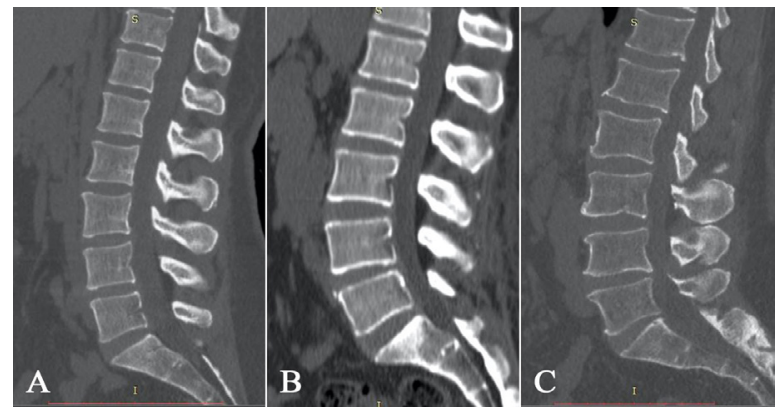

Figure 6 . Postoperative $6^{\text {th }}$ month sagittal computed tomography images of the patients due to far lateral lumbar disc herniation (FLLDH). Patients operated for left (A) L2-3, (B) left L4-5 and (C) right L3-4 FLLDH that postoperative severe burning dysesthesia in FLLDH was due to the traction of spinal nerve during dissection. Moreover, O'Hara and Marshall(19) reported that earlier visualization of posterior ramus and secure dissection of the extraforaminal area reduced the risk of postoperative sensory deficit. We also managed to reduce the risk of postoperative sensory deficit by exposing the exiting nerve root earlier (Figure 4).

There are various approaches for the surgical treatment of FLLDH: medial facetectomy, full facetectomy, intertransverse approach, percutaneous endoscopic approach, anterolateral retroperitoneal approach and lateral extraforaminal approach. Comparing lateral and medial approaches has revealed more satisfactory results with lateral approaches ${ }^{(4,20)}$.

However, since the anatomical landmarks and route that is followed are not entirely known in lateral approach, medial approach is preferred more often. In a study comparing the different surgical procedures, Epstein ${ }^{(4)}$ obtained better results with the lateral approach. In addition, Ryang et al. ${ }^{(20)}$ reported excellent results in \%95 of cases using lateral approach while $57 \%$ in medial approach. O'Hara and Marshall(19) reported good and excellent results at the postoperative $14^{\text {th }}$ month follow-up in $90 \%$ of 20 patients operated using the lateral approach. In their study, Marquardt et al.(7) reported excellent outcomes in $75.9 \%$ and good outcomes in $18.4 \%$ in the long-term followup of patients, who were operated via minimally invasive lateral approach. In addition, Porchet et al. ${ }^{(21)}$ reported good and excellent results in $73 \%$ of cases using a microsurgical far lateral approach while Weiner and Dabbah ${ }^{(22)}$ reported the same outcomes in $85 \%$ of their cases. Similarly, Sasani et al.(23) obtained good and excellent results using a lateral endoscopic approach in $86.4 \%$ of their patients. In our study, using lateral interpedicular approach without exposing the intertransverse

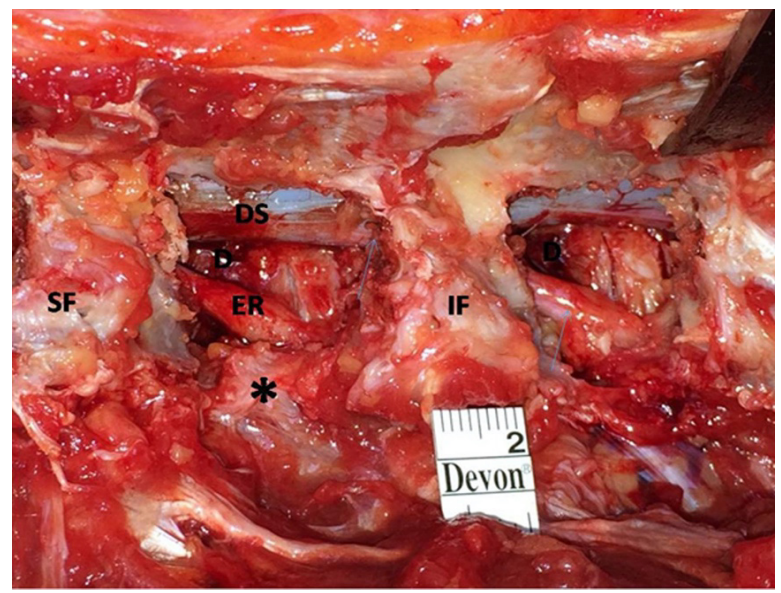

Figure 7. A picture from fresh cadaveric dissection. Pars interarticularis is completely removed for understanding of the relationship of exiting and traversing nerve roots and intervertebral disc, and inferior facet joint and pars interarticularis

SF: Superior facet joint, IF: Inferior facet joint, blue arrow: Traversing root becomes exiting root at the lower level, ER: Exiting nerve root, DS: Dural sac, *: Intertransverse muscle and fascia 
fascia, we obtained excellent results in $78.5 \%$ of our cases and good results in $14.2 \%$ based on the MacNab classification. Epsteins reported that the intertransverse fascia was exposed in the muscle splitting approach he described, while Schlesinger et al. ${ }^{(18)}$ exposed the intertransverse fascia in their lateral microsurgical approach (4). O'Hara and Marshall(19) also reported that the intertransverse fascia was exposed in the new muscle splitting approach he described. Salame and Lidar $^{(2)}$ reported that the intertransverse fascia was opened in a minimally invasive technique using METRx tissue dilators. Also, Tessitore reported that the intertransverse muscle was opened in a microsurgical transmuscular approach, while Ryang et al.(20) reported that the intertransverse fascia was opened using a lateral transmuscular approach ${ }^{(5)}$. The intertransverse ligament was released in the paramedian approach that was used by Park et al.(16) . In the lateral interpedicular technique we used, intertransverse fascia exposure and visualization of the lateral facet and transverse process were not required. Since the nerve root was exposed at the location where it exited the medial aspect of the pedicle located superiorly, no complications of exiting root injury were observed.

It is possible to reach the foraminal and extraforaminal zones by using the lateral interpedicular technique. The herniated disc material can be reached with limited bone removal, which leads to exposure of compressed nerve root and dorsal root ganglion and by the way, does not cause instability. When compared to medial approach, which requires excessive bone removal, the risk of instability is minimal due to minimal bone resection. The lack of need for opening the intertransverse fascia and exposing the lateral aspect of the facet joint and transverse processes minimizes muscle retraction and hemorrhage at the surgical site and shortens the duration of the surgery. Studies comparing the lateral versus medial approaches reported more satisfactory results with a lateral approach ${ }^{(6,20)}$. However, since the anatomical landmarks and the route followed are not fully identified in lateral approaches, the medial approach is resorted to more often. Future experience and relevant anatomical studies on cadavers may allow the more frequent use of lateral approaches, with a better understanding of the extraforaminal zone anatomy.

\section{Study Limitations}

Some limitations existed in our study. Firstly, intertransverse ligament is not so much functionally important anatomical structure but there are many vessels beneath the ligament. The blood supply to the root has been shown to be critically dependent on the lateral radicular vessels ${ }^{(24,25)}$. Therefore, ischemic changes due to the disc fragment compression may be the cause of the acuteness of the symptoms seen in FLLDHs. So, we did not need to open the intertransverse ligament and therefore, we avoided bleeding and using bipolar cautery. Secondly, our study, consisting of only 28 patients, was relatively small. Also, the retrospective nature of this study hindered prospective analysis and randomization. In order to determine the clinical predictive value of superior articular process excision without opening intertransverse ligament, long-term follow-ups and large-scale prospective studies are required. Finally, we could achieve postoperative computed tomographies only six months after the operation; therefore, we need a longer follow-up period to evaluate the results, especially iatrogenic instability.

\section{CONCLUSION}

The LISA without exposing the intertransverse fascia enables direct access to migrated or non-migrated far lateral disc herniations and to preserve facet joint and pars interarticularis functionally. Additionally, minimal bone removal of the superior articular process of the facet provides a better exposure of disc space. It prevents excessive muscle retraction since there is no need to expose lateral aspect of the facet joint and transverse processes. Moreover, it avoids the risk of neurological damage by enabling the identification of the exiting nerve root in the early phase of the operation. Overall, the lateral interpedicular approach is a safe technique with a relatively low complication rate, associated with less tissue damage. It is a minimally invasive procedure when compared to remaining medial and lateral approaches and requires less bone removal.

\section{Ethics}

Ethics Committee Approval: This study was approved by The Council of Forensic Medicine (decision no: ATK 0.01.00.08/74). Informed Consent: Informed consent was obtained from the patients.

\section{Authorship Contributions}

Surgical and Medical Practices: A.K., H.C., Concept: A.K., H.C., Design: A.K., H.C., Data Collection or Processing: A.K., H.C., Analysis or Interpretation: A.K., H.C., Literature Search: A.K., H.C., Writing: A.K., H.C.

Conflict of Interest: No conflict of interest was declared by the authors.

Financial Disclosure: The authors declared that this study received no financial support.

\section{REFERENCES}

1. Eicker SO, Rhee S, Steiger HJ, Herdmann J, Floeth FW. Transtubular microsurgical approach to treating extraforaminal lumbar disc herniations. Neurosurg Focus. 2013;35:E1.

2. Salame K, Lidar Z. Minimally invasive approach to far lateral lumbar disc herniation: technique and clinical results. Acta Neurochir (Wien). 2010;152:663-8

3. Cervellini P, De Luca GP, Mazzetto M, Colombo F. Micro-endoscopicdiscectomy (MED) for far lateral disc herniation in the lumbar spine. Technical note. Acta Neurochir Suppl. 2005;92:99-101.

4. Epstein NE. Foraminal and far lateral lumbar disc herniations: surgical alternatives and outcome measures. Spinal Cord. 2002;40:491-500.

5. Tessitore E, de Tribolet N. Far-lateral lumbar disc herniation: the microsurgical transmuscular approach. Neurosurgery. 2004;54:939-42. 
6. Epstein NE. Evaluation of varied surgical approaches used in the management of 170 far-lateral lumbar disc herniations: indications and results. J Neurosurg. 1995;83:648-56.

7. Marquardt G, Bruder M, Theuss S, Setzer M, Seifert V. Ultra-long-term outcome of surgically treated far-lateral, extraforaminal lumbar disc herniations: a single-center series. Eur Spine J. 2012;21:660-5.

8. Samini F, Bahadorkhan G, Ehsaei MR, Kheradmand H. Intraforaminal and extraforaminal far lateral lumbar disc herniation (a review of 63 cases). Med J Islam Repub Iran. 2008;22:63-7.

9. Postacchini F, Montanaro A. Extreme lateral herniations of lumbar disks. Clin Orthop Relat Res. 1979;222-7.

10. Fankhauser H, De Tribolet N. Extraforaminal approach for extreme lateral lumbar disc herniation. Operative spinal surgery Churchill Livingstone, Edinburgh. 1991:145-60.

11. Fankhauser $\mathrm{H}$, de Tribolet $\mathrm{N}$. Extreme lateral lumbar disc herniation. $\mathrm{Br}$ J Neurosurg. 1987;1:111-29.

12. O’Toole JE, Eichholz KM, Fessler RG. Minimally invasive far lateral microendoscopic discectomy for extraforaminal disc herniation at the lumbosacral junction: cadaveric dissection and technical case report. Spine J. 2007;7:414-21.

13. Viswanathan R, Swamy NK, Tobler WD, Greiner AL, Keller JT, Dunsker SB. Extraforaminal lumbar disc herniations: microsurgical anatomy and surgical approach. J Neurosurg. 2002;96(2 Suppl):206-11.

14. Macnab I. Negative disc exploration. An analysis of the causes of nerve-root involvement in sixty-eight patients. J Bone Joint Surg Am. 1971; 53:891-903.

15. Abdullah AF, Ditto EW 3rd, Byrd EB, Williams R. Extreme-lateral lumbar disc herniations. Clinical syndrome and special problems of diagnosis. J Neurosurg. 1974;41:229-34.

16. Park HW, Park KS, Park MS, Kim SM, Chung SY, Lee DS. The Comparisons of Surgical Outcomes and Clinical Characteristics between the Far Lateral Lumbar Disc Herniations and the Paramedian Lumbar Disc Herniations. Korean J Spine. 2013;10:155-9.

17. Porchet $F$, Fankhauser $H$, de Tribolet $N$. Extreme lateral lumbar disc herniation: clinical presentation in 178 patients. Acta Neurochir (Wien). 1994;127:203-9.

18. Schlesinger SM, Fankhauser $H$, de Tribolet N. Microsurgical anatomy and operative technique for extreme lateral lumbar disc herniations. Acta Neurochir (Wien). 1992;118:117-29.

19. O'Hara LJ, Marshall RW. Far lateral lumbar disc herniation. The key to the intertransverse approach. J Bone Joint Surg Br. 1997;79:943-7.

20. Ryang Y, Rohde I, Ince A, Oertel M, Gilsbach J, Rohde V. Lateral transmuscular or combined interlaminar/paraisthmic approach to lateral lumbar disc herniation? A comparative clinical series of 48 patients. J Neurol Neurosurg Psychiatry. 2005;76:971-6.

21. Porchet F, Chollet-Bornand A, de Tribolet N. Long-term follow up of patients surgically treated by the far-lateral approach for foraminal and extraforaminal lumbar disc herniations. J Neurosurg. 1999;90(1 Suppl):59-66.

22. Weiner BK, Dabbah M. Lateral lumbar disc herniations treated with a paraspinal approach: an independent assessment of longer-term outcomes. J Spinal Disord Tech. 2005;18:519-21.

23. Sasani M, Ozer A, Oktenoglu T, Canbulat N, Sarioglu A. Percutaneous endoscopic discectomy for far lateral lumbar disc herniations: prospective study and outcome of 66 patients. Minim Invasive Neurosurg. 2007;50:91-7.

24. Wang Q-p, Lee N-s, Zhang Y, Liu J, Zhu J-y. Intertransverse approach for extraforaminal herniations. Spine (Phila Pa 1976). 1997;22:701-5.

25. Owen JH, Naito M, Bridwell KH, Oakley DM. Relationship between duration of spinal cord ischemia and postoperative neurologic deficits in animals. Spine (Phila Pa 1976). 1990;15:618-22. 